EPJ manuscript No.

(will be inserted by the editor)

\title{
Localized Structures in Convective Experiments
}

\author{
Javier Burguete, Héctor Mancini \\ Depto. Física y Matemática Aplicada, Universidad de Navarra, Irunlarrea 1, E-31008 Pam- \\ plona, Spain
}

\begin{abstract}
In this work we review localized structures appearing in thermo-convective experiments performed in extended (large "aspect ratio") fluid layers. After a brief general review (not exhaustive), we focus on some results obtained in pure fluids in a Bénard -Marangoni system with non-homogeneous heating where some structures of this kind appear. The experimental results are compared in reference to the most classical observed in binary mixtures experiments or simulations. In the Bénard-Marangoni experiment we present the stability diagram where localized structures appear and the typical situations where these local mechanisms have been studied experimentally. Some new experimental results are also included.
\end{abstract}

\section{Introduction}

From the experimental point of view and considering the term in a wide sense, a "localized structure" can be observed as a fixed (or drifting) spatially limited dynamic structure, overlapped on a different and extended dynamic state (homogeneous or patterned). To our knowledge, the name was used for the first time in [1]. In 1993 Cross and Hohenberg [2] defined this kind of structure, as a local perturbation appearing in systems by them called of "Type Io" (i.e.,wave number qo $\neq 0$, and frequency $w o \neq 0)$. This situate "localized structures" in the frame of instabilities associated to a structure normally embedded in an otherwise ideal pattern, formed by traveling waves.

However, in some previous experiments other local confined states immersed in extended patterns has been named "coherent structures" (bubbles, thermal plumes, swirls, etc). Coherent structure in this frame (principally in turbulent flows and in boundary layers) is any local structure with a lifetime comparable or higher to the transit time through the container [3]. That is, a local structure that keeps its shape at least during a characteristic transit time in the planform. If the spatial perturbation of the base state is stationary it could be considered as a "local defect" in a patterned plane, (even if it is traveling mounted on a mean flow of the homogeneous state). This kind of patterns appear normally on homogeneous states with linear traveling waves or feebly non-linear waves.

In this brief review we are focusing on the first kind of localized states. That is, a local convective structure with a different dynamic from the basic state. The basic

Send offprint requests to: hmancini@unav.es 


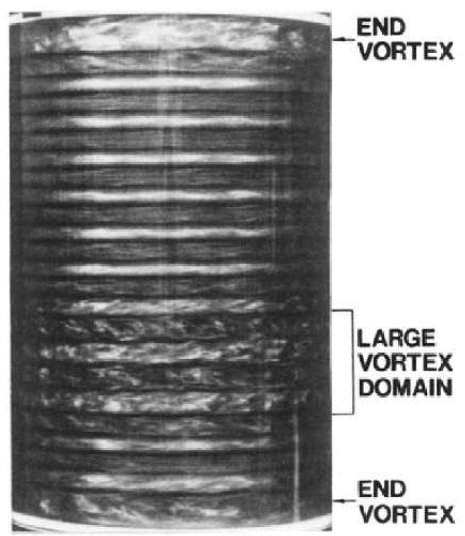

Fig. 1. Localized structure inside a periodic stationary pattern in a Taylor-Couette flow (from [18]).

state could be homogeneous (conductive), traveling waves, rolls, hexagons, or even chaotic, and usually, the local structure interact with it, being confined or acting as a source/sink for new traveling waves. Higher order non linearities play a basic role in this kind of states. Localized structures in thermo-convective flows have been studied both experimentally and numerically in different systems since many years ago. Convection in fluid layers of binary mixtures heated from below, in containers with different lateral geometry like rectangular [4-6], cylindrical[7], or annular $[1,9]$ has been some of the key experiments performed in the first stage.

Convective systems with other kind of fluids and geometries have also been studied experimentally. Localized traveling rolls have been shown in experiments of convection in nematic liquid crystals under electric fields [10], in cryogenic $3 \mathrm{He}-4 \mathrm{He}$ binary mixtures and even in pure fluids with inhomogeneous heating [11]. Also has been studied numerically in magnetofluids (where the name "convecton" appear for the first time) $[12,13]$, in $3 \mathrm{He}-4 \mathrm{He}$ mixtures $[14,15]$, and in Marangoni convection (surface tension driven) in binary fluids [16], but the number of new successful experiments reported up today is very low.

We would like also to mention that other kind of localized solutions have been reported in different fluid experiments. For instance, the existence of turbulent spots on top of laminar solutions, as appear in the transition to turbulence in Rayleigh convection [17]. There is also an analogy between convective systems and Taylor Couette experiments, where the driving force is inertia instead of the Archimedean force. Turbulent spots have also been reported (fig.1) in these experiments [18]. Other experiments with similar behavior are plane Couette-flow one's [19], or pipe flow turbulent spots [20]. In this work, we will focus on laminar localized structures.

Localized structures have been related from the beginning to solutions obtained in the frame of Ginzburg Landau equations [21], and since then, a lot of mathematicalnumerical work was done, even without an explicit reference to any experiment. Calculations anticipated results that in many cases have been after confirmed in experiments, as the "hole" type solutions $[22,23]$. More recently, another group of numerical studies with Ginzburg-Landau equations including higher order non-linearities (quintic) like in ref. [24-28] among others, describing very interesting localized solutions that to our knowledge, still remain without an experimental counterpart, open the door to perform new experiments. 


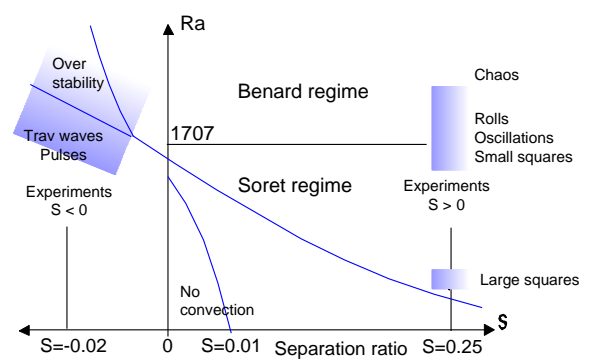

Fig. 2. Parameter region to obtain localized structures in binary mixtures heated from below. The corresponding experiments are for $S<0$, references $[1,6,7,9]$ and for $S>0$, reference [8].

\section{Convection in binary mixtures}

Thermoconvection in binary fluids heated from below is a well studied example of "Type Io" system [2], where time and space scales are similar to the Bénard-Rayleigh convection in pure fluids[29] under the same driving forces. We remember here that Bénard-Rayleigh convection (without surface tension) occurs in a fluid between two conductive plates and convection is driven by buoyancy forces. The first pattern that appear (for high Prandtl fluids) is normally a set of parallel rolls. With the upper surface open to the air, surface tension add a cooperative gradient and the basic pattern becomes composed by hexagonal cells. In binary mixtures a concentration gradient (horizontal) appears, and there is a possibility to have a first bifurcation oscillatory (when separation ratio is negative), a fact that immediately brings to have small amplitude traveling waves and a very rich phenomenology in the planform. It is well known that under this condition (negative separation ratio) besides the Hopf bifurcation other two features, a subcritical bifurcation to convection and a dependence on a other parameters like a small parameter, the Lewis number, complicates the behavior.

The main differences in binary mixtures are additional effects due to concentration gradients creating a solute flow due to Soret effect, and a separation in the time scales controlled by Lewis number. The new relevant parameters are: the Rayleigh number Ra, Soret number, Prandtl number Pr, the separation rate S, and the mentioned Lewis number L.

Rayleigh number is a measure for relative importance of destabilizing and stabilizing buoyancy effects and usually in experiments it is the main control parameter. Prandtl number is the ratio of characteristic thermal and viscous relaxation times, and define the type of fluid used $(\operatorname{Pr}=\nu / k$, where $\nu$ is the viscosity and $\mathrm{k}$ the thermal diffusivity). It is important for dynamical behavior and non-linear regimes after secondaries instabilities (the conductive-convective threshold is independent of $\mathrm{Pr}$ ). A high Prandtl number fluid (higher than 10)is better to have separated the inertial and buoyancy regimes. The Lewis number, that separate the time scales, is the ratio between time scales for thermal and solute diffusion respectively $(L<<1)$.

Linear stability analysis of the thermal conducting state divide roughly in two main groups the convective patterns obtained in the experiments. Depending mainly, on the separation rate $\mathrm{S}$ sign (positive or negative), and providing that $\mathrm{L}$ is small, localized structures appear usually for $\mathrm{S}$ negative (see Figure 2). A positive separation rate $(S>0)$ means a higher concentration of the less density component in the bottom part of the container (unstable stratification)and brings to a different situation. For negative separation rates, oscillations are due to the difference between diffusion times for heat and concentration, the last acting as "elastic force". It should be noted that 


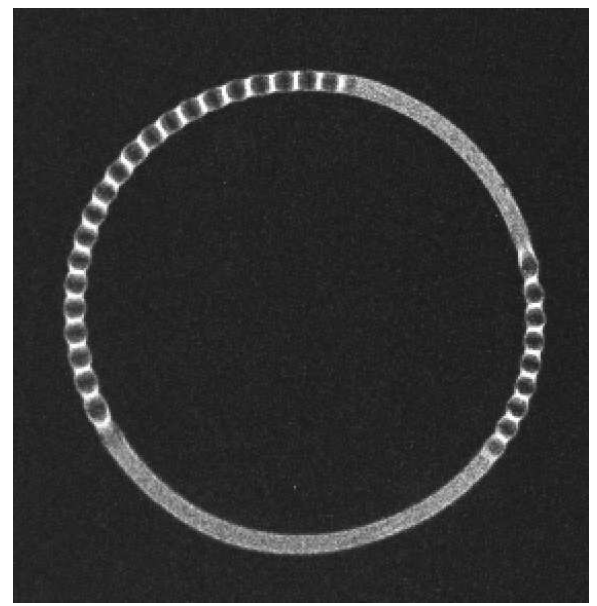

Fig. 3. Localized structures in binary mixture convection in an annular configuration (P. Kolodner, unpublished, private communication).

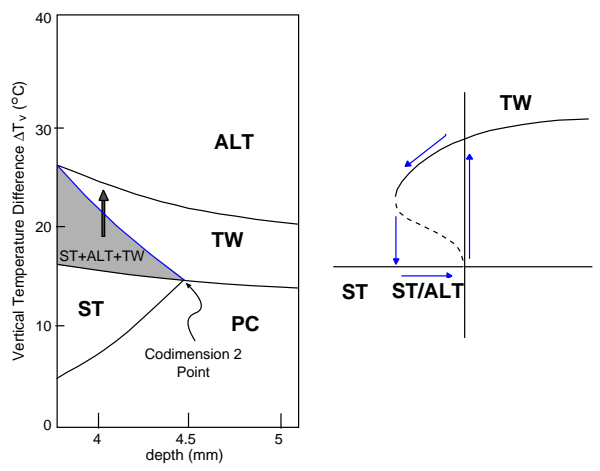

Fig. 4. Left: Simplified stability diagram in a one-dimensional heating experiment. Stationary patterns have been named ST (cellular pattern), PC (primary convection). Two time dependent patterns can be distinguished: pure traveling waves (TW), and a mixed state of both traveling waves and the stationary cellular pattern (ALT). Right: Sketch of the subcritical bifurcation in the gray marked region, where the control parameter increased is the temperature difference. The size of the subcritical region is very large, compared to other experimental setups.

it means an small amplitude instability. Strong movements destroy the concentration effects. The role played by the concentration field, among others analysis, can be found in references [30-34].

The results of the experiment have been early related to solutions in complex Ginzburg-Landau equations. A general theoretical 3D approach to convection in binary mixtures was presented in [35], oriented to positive separation ratio S.

Experiments have been performed, both for positive and negative separation ratio, mainly in two components mixtures of ethanol and water, but also in more sophisticated cryogenics mixtures like $3 \mathrm{He}-4 \mathrm{He}$. To our knowledge, localized structures in convective experiments have been reported for the first time as appearing in these systems [1]. 


\subsection{Negative Soret Coefficients}

As it was said, convection appear as growing oscillations which evolve into standing waves when separation rate is negative. With increasing amplitude these waves undergo a transition to traveling waves, and then to more complex waveforms. Out of these states emerge stable stationary spatially localized structures embedded in a background of small amplitude standing waves (see fig- 3). In 1990 P. Kolodner [36, 9,37-39], presented evidence for the reflection of traveling waves in a localized structure. The relation of these states to the time-independent spatially localized states that characterize the so called pinning region is investigated by exploring the stability properties of the later, and the associated instabilities. The system studied by them differs from both binary fluid convection and natural doubly diffusive convection, although like the others, it is reversible in space and non-variational [16]. Specifically, the time-independent binary fluid system studied in $[47,52]$ is equivariant under the spatial reflection $\mathrm{R}$ : $(x)$ to $(-x)$, where $(x)$ is the horizontal coordinate, and hence is reversible as dynamical system in space, i.e. $(x)$ acts as time-like variable $[14-16,42$, 46-56].

\section{Localized structures in pure fluids: non-homogeneous heating}

The first experiments in the study of thermoconvective localized structures, were performed almost exclusively in binary fluids. However, localized structures have been reported also in pure fluids with lateral heating, or non-homogeneous systems heated from below. In both systems, convection was studied with the upper surface of the fluid open to the air, that is, including both buoyancy and surface tension forces (Bénard- Marangoni convection). Also in both systems the existence of an additional horizontal gradient could play the role of the concentration gradient in binary mixtures.

Localized structures have been reported in experiments [11],[44], [57] and in simulations in $[58,59]$. During 90's years, experimental interest in this kind of systems was focused in other problems like to classify patterns, quantify effects of boundaries (geometry and properties), transitions between patterns or evolution of defects. To our knowledge, in these experiment reports on localized structures are rather scarce and mixed with other results.

The quasi-unidimensional system on which the results presented here have been obtained, was described initially in [45] and in many other papers from the same authors. It is a variation of former experiments developed with a hot wire near the surface of a fluid [43]. To put order in the richness of all experimental situations obtained was a more than 10 year task. The first stability diagram appears in [11], and more complete stability diagrams where presented in $[63,65]$. We have presented a simplified version of these diagrams in figure 4, where bifurcations with conditions to produce localized structures have been identified (vertical arrow) as a function of the fluid layer depth.

The main characteristic of this experiment is that all the localized structures that we will present here appear on the top of a patterned stationary structure. Increasing the control parameter other solutions (traveling waves) can appear through a subcritical instability. Most of the results that we are going to discuss appear in the region where the arrow is plotted in figure 4.

The experimental setup of these experiments (see fig.5), essentially consist on a fluid layer placed in a narrow channel with the surface open to the atmosphere, and where the temperature of the bottom plate is not homogeneous. A nearly Gaussian profile is created along the shorter dimension $(y)$, and consequently we have combined 
(a)

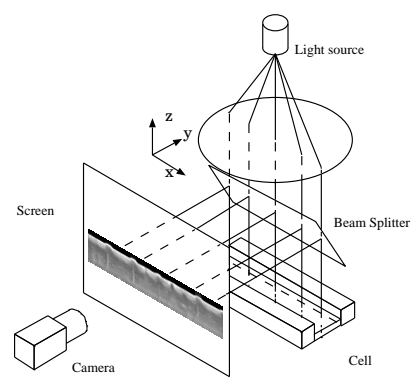

(b)

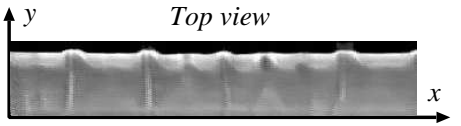

$t=\mathrm{T}$

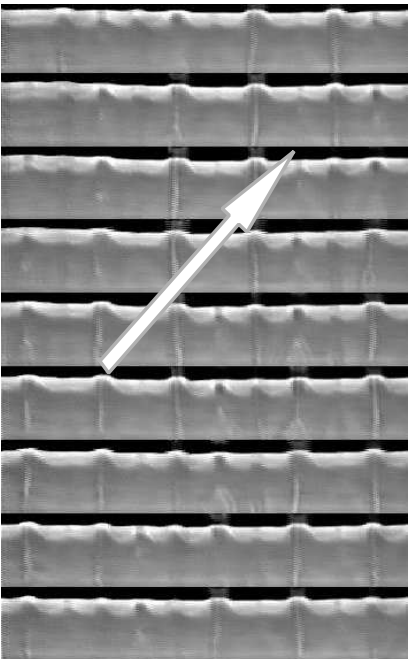

(c)

Fig. 5. Top left: Experimental setup where the convective channel and the shadowgraph system are presented. A sample of the observed $2 \mathrm{~d}$ pattern is presented in the screen. Bottom left: Characteristic 2D pattern. The convective cellular pattern can be observed in the $x$ direction. Only one half of the experimental cell is shown for the shake of simplicity. The other half will appear reflected on the $y$ direction. Right: Sequence of snapshots, where the existence of localized propagative structures can be observed.

two effects (i) a classical Bénard-Marangoni convection due to the vertical gradient applied, where a threshold must be crossed to produce convection and (ii) a lateral heating convection instability where the horizontal temperature gradient destabilizes the fluid without threshold (wavenumber $k=0$ ). A detailed description on the different experimental setups that produce this dynamics can be found in $[11,63,64]$. This experiment has also been used to analyze the defects dynamics that can be frozen depending on how the control parameter is increased (change rate) when crossing the threshold [65-67].

As a result, the dynamics of the different patterns is very rich, and some of the states presented here have been found in lateral heating experiments for large depths [68] and even in Taylor-Dean experiments [60]. The primary state is a couple of homogeneous counterrotating rolls whose axis is parallel to the longest dimension $(x)$ of the cell. When the vertical temperature gradient (a control parameter) is further increased, this roll will break its translational symmetry in (i) a stationary cellular pattern for small depths (ST), or (ii) a traveling cellular pattern for large depths (TW). These two solution coexist in a Codimension 2 point, that separates both regimes (see fig. 4, left). Here we will focus in a position close but below this point (where the black arrow is presented).

Over the cellular stationary pattern (ST) a secondary subcritical instability can appear, and two symmetrical traveling solutions are created on top of the stationary pattern (ALT). Depending on the control parameters and on the short term history of the experiment different situations can be reached. Concerning localized structures, we can differentiate two groups: (i) perturbations of the basic state and (ii) localized structures of one of the solutions on top of other of the patterns.

In the following, we will discuss these localized structures. 

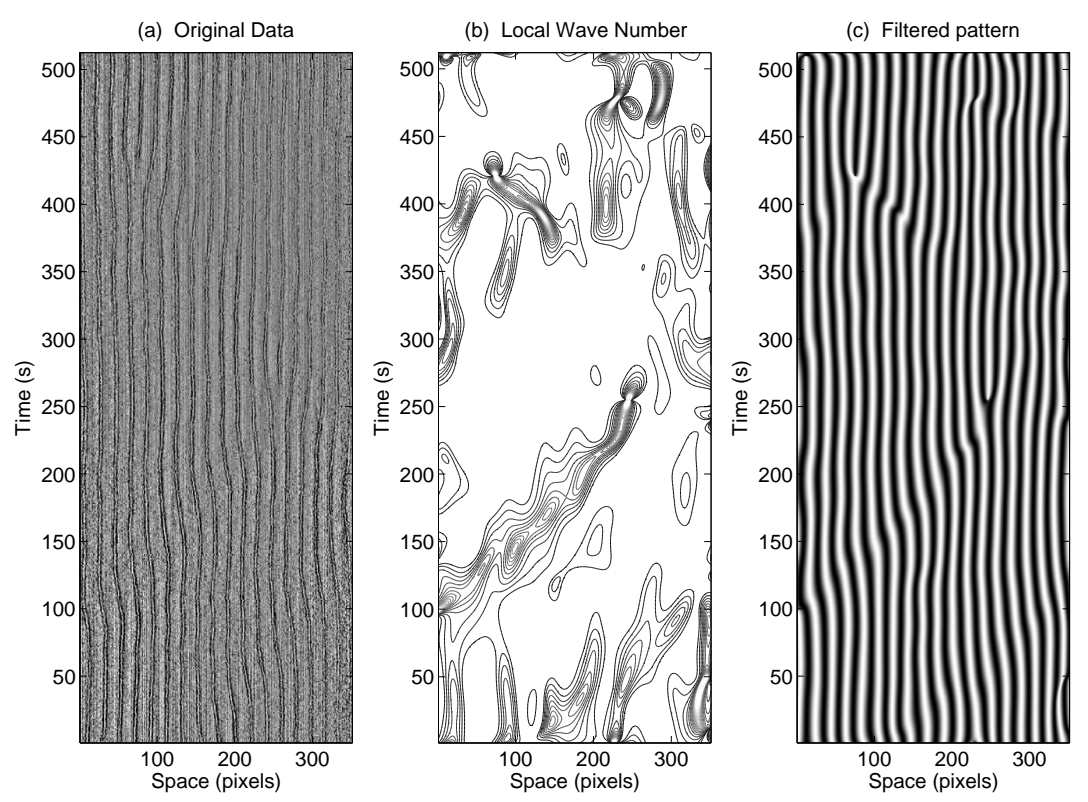

Fig. 6. Phase soliton in a pure fluid convective experiment. Left: original spatio-temporal pattern (from [11]). Middle: local wavenumber. A propagative pulse can be observed to the right. Right: filtered pattern where the phase modulation of the stationary pattern is more visible.

\subsection{Localized phase pulses}

This situation appears just below the threshold of the gray region where the three regimes can appear simultaneously (marked as ST+ALT+TW in fig. 4). These localized structures are localized states in the sense we can define a region where a perturbation exists for times longer than the typical experimental times. In figure 6 we present one of the typical behaviors observed below the threshold. Over the stationary pattern there is a perturbation of the phase of the stationary pattern that propagates with a very well defined velocity (non-linear group velocity). This perturbation can be reflected at the lateral wall of the experiment, and can even collide with other pulses. One of these collisions appear around $t=250$, for $x=250 \mathrm{px}$. The right-coming pulse is difficult to see. As a consequence, one of the pulses disappears and only one, traveling to the left, survives.

In the middle panel of figure 6 we plot the local wavenumber, where these perturbations are clearly visible. Instead of the situation of NB holes $[22,23]$ these pulses are no associated with a traveling hole of the amplitude of a propagating wave. These last solutions can be modeled and described with high accuracy using a G-L model. Here only on the vicinity of the spatio-temporal defects where the collision takes place, the amplitude of the stationary pattern vanishes. In other places the amplitude fluctuates, but around a constant value. This kind of structures were predicted in refs [61, $62]$. 
(a) Original Image

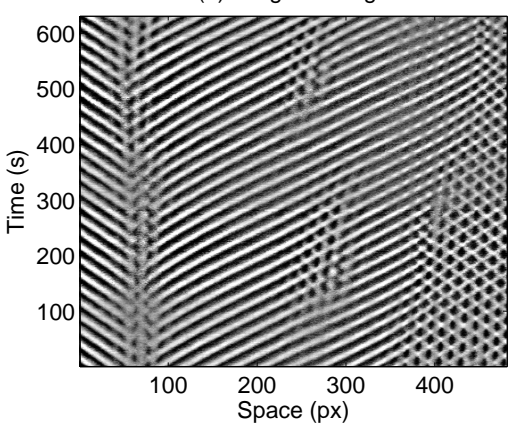

(c) Localized states (TW2)

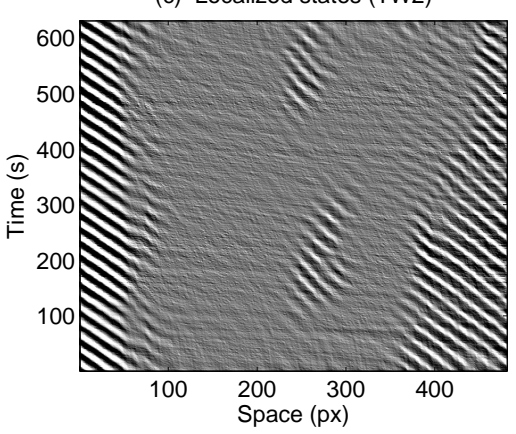

(b) Localized states (Stationary Mode)

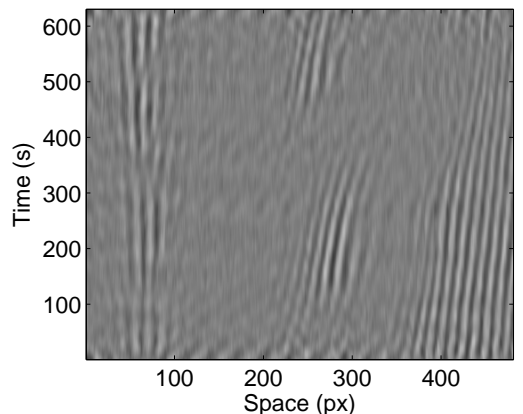

(d) Base state (TW1)

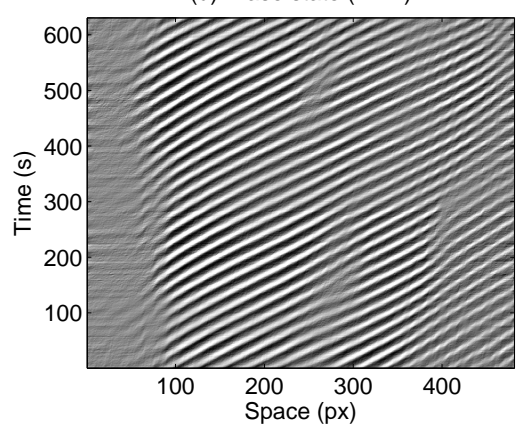

Fig. 7. Spatio-temporal diagrams for a transient localized structure over a TW. (a) Original spatiotemporal diagram (from [57]. (b) and (c) localized structures corresponding to the new states ST and TW2. (d) Base state TW1 that supports the localized structures.

\subsection{Localized states over extended solutions}

If we further increase the control parameter (the vertical temperature difference $\Delta \mathrm{T}_{v}$ ), new modes appear on the system. These modes are propagative, instead of the one that constitutes the stationary base state (ST). Moreover, the wavenumber of these propagative modes (in the following, TW1 and TW2, depending on the propagation direction, towards positive or negative $x$ values) is one half of the wavenumber of the stationary pattern. As it was stated above, if we increase the control parameter $d$, we will cross through a codimension- 2 point, and from that point the TW appears over an homogeneous state.

When the threshold of these propagative states is crossed, three different solutions can coexist on the system, each one of them is stable, although the stability of each one of these states depends on the experimental parameters. Here we will focus on two situations: (i) a transient localized solution that appears on the top of traveling waves (fig. 7) and (ii) stable localized solutions that appear on the top of stationary waves (fig. 8).

\section{Transient localized structures}

One of the possible states the system can reach is a source of traveling waves localized somewhere along the channel. On top of these propagative solutions we may find 

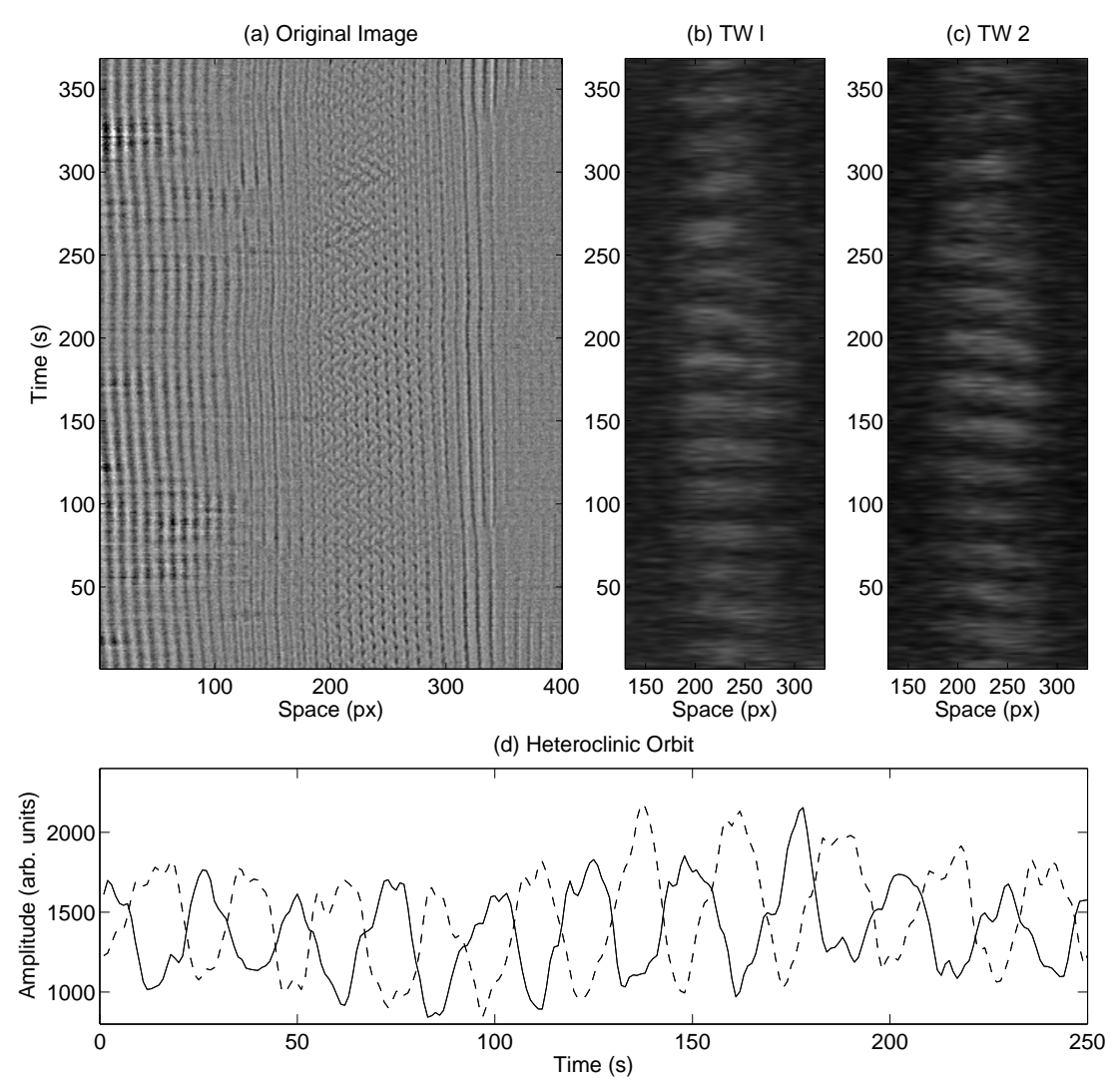

Fig. 8. Pulsating localized pattern. Top left: Original spatiotemporal diagram (from [57]). Top middle and right: filtered amplitudes for the traveling waves TW1 and TW2 between $150<x<350 \mathrm{px}$. The amplitude of these modes alternate periodically. Bottom: average amplitude of each TW. A heteroclinic orbit can be identified between both solutions.

different regions where the other solutions appear: Localized structures of the two other modes (ST and the other TW) can be identified. These solutions exist for short periods of time, and indicates the strong non-linear coupling that exists between the different modes. Inside each one of these localized structures the TW are very well defined (wavenumber and amplitudes), but there is a modulation on the stationary mode (the associated wave number is not constant, but decreases in the direction where the base state propagates). Also, in these solutions a defect appears at the core of these solutions, simultaneously in both solutions TW1 and TW2.

We would like also to note that in this situation the core of the source of waves is controlled by the presence of a localized state of the stationary mode. In some way, this stationary mode couples the two propagative solutions that growth to different spatial domains. 


\section{Stable localized structures}

As discussed in detail in $[63,65]$, the bifurcation towards these new waves is subcritical, and consequently there is a hysteresis region. Different spatial configurations can appear, depending on the history of the experiment. For some choice of parameters, the stationary pattern wins, and in other cases a very rich dynamics can be triggered. But somewhere in this hysteresis region all the solutions will share a similar stability, and the fronts that separates the solutions should remain stationary (Maxwell point).

In this configuration there is a whole range of values of the control parameter where localized states with stable boundaries are found. In this region we have typically both traveling waves that are fired simultaneously, living on top of the stationary cellular pattern, the alternating pattern ALT. For some parameter values we find islands of this alternating pattern surrounded by stationary pattern (not shown here, but can be observed, for example in $[63,65]$ ). These results have been reproduced in numerical simulations by [28] and are similar to those proposed as a result of snake-bifurcations $[48,16,49]$. In those bifurcations, a special localized solution appears around the Maxwell point in the bistable region of the parameter space. The spatial extension of these solutions depend on which one of the stable branches is selected. The fronts are stable, and the system can increase or decrease its size jumping between stable branches for the same parameter values.

But an even more interesting dynamical behavior appears for larger values of the control parameter. In that case, there is a competition between the stability of the two traveling solutions, that now are not combined but pulsate alternatively. In figure 8 we plot one of this states. In fig. 8(a) we present the original pattern, where the localized structure can be observed in the spatial region ranging from $x=200$ to $300 \mathrm{px}$. The stationary pattern is always present, and in top of it both traveling solutions appear. The amplitude of each one of these solutions is presented in figures 6.b and 6.c. Black means zero amplitude, and white the maximum value. The TW1 (resp. TW2) identifies the left (resp. right) traveling wave. The maximum amplitude is reached alternatively between both solutions. To emphasize this fact, the average amplitude is presented in the bottom panel of fig. 8 . The dashed line corresponds to the right TW, and the solid line to the left TW. A heteroclinic cycle can be identified between both solutions. A similar behavior can be reproduced using Quasi1D solutions [69].

\section{Conclusions}

We have presented a quick review of the experimental localized states that can be found in convective systems, where buoyancy plays an important role destabilizing the originally stationary fluid layer. These states can appear in binary mixture convection but also in pure fluid convection. The first configuration is the original setup where these states where described, and the last constitutes a new approach to study these objects. In both systems, there are at least three different physical mechanisms in competition, but the details differ. In binary mixtures the key point is the competition between the two species, and how these concentration gradients interact with the temperature gradients. As a result, stationary or propagating localized states can be found.

In pure convective experiments, localized structures can be observed provided that two different thermal instabilities appear combined. Here we have presented the case of a system where horizontal and vertical temperature gradients are applied simultaneously, each one giving rise to a different instability (Bénard-Marangoni and lateral heating convection). In Bénard-Marangoni the first solution is a stationary pattern, whereas for lateral heating the basic solution are propagative waves. When 
these two effects are combined, there is a region where these two solutions compete, and localized solutions can be created. We have summarized the main situations that can be measured, and we have presented new dynamical mechanisms between these patterns.

\section{Acknowledgments}

The authors want to honor Prof. H. Brandt in his 60th. birthday and to thank him for helpful discussions. We thank also Prof. E. Knobloch, Prof. I. Mercader and Prof. O. Descalzi for useful help with the bibliography on the subject. We especially would like to thank M. Miranda who performed many of the experiments presented here. This work has the financial support from Spanish Ministry of Science and Technology under Contract No. FIS2011-24642.

\section{References}

1. J.J. Niemela, G. Ahlers, D. Cannell, Phys. Rev. Lett. 64 (1990) 1365.

2. M. C. Cross and P. C. Hohenberg Reviews of Mod. Phys. 65 (1993) 851-1112.

3. G. Zocchi, E. Moses, A. Libchaber, Physica A 166 (1990) 387-407.

4. E. Moses, V. Steinberg, Physical Rev. A, Vol.34 (1986) 693-696.

5. R. Heinrichs, G. Ahlers, D.S. Cannell, Phys. Rev. A 35 (1987) 2761.

6. V. Steinberg, E. Moses, J. Fineberg, Nucl. Phys. B 2 (1987) 109.

7. E. Moses, J. Fineberg, V. Steinberg, Phys. Rev. A 35 (1987) 2757.

8. E. Moses, V. Steinberg, Phys. Rev. A 43 (1991) 707.

9. D. Bensimon, P. Kolodner, C.M. Surko, H. Williams, V. Croquette, J. Fluid Mech. 217 (1990) 441.

10. A. Joetz and R. Ribota, in Propagation in Systems far from Equilibrium J.E. Wesfreid, H.R. Brandt,P. Manneville, G. Albinet,N. Boccara (Edts.)(Springer-Verlag, Berlin 1987)194-203

11. J. Burguete, D. Maza, H. Mancini, Physica D 174 (2003) 56-70.

12. S. Blanchflower, Phys. Lett. A 261 (1999) 74-81.

13. S. Blanchflower, N. Weiss, Phys. Lett. A 294 (2002) 297-303.

14. O. Batiste, E. Knobloch, Phys. Rev. Lett. 95 (2005) 244501-1/4.

15. O. Batiste, E. Knobloch, Physics of Fluids 17 (2005) 064102-1/14.

16. P. Assemat, A. Bergeon, E. Knobloch, Phys. Rev. A, 76, (2008) 693-696.

17. F. Daviaud, M. Bonetti, M. Dubois, Solitons and Chaos, Research Reports in Physics (Springer, Berlin, 1991) 129-134.

18. G.W. Baxter, C.D. Andereck, Phys. Rev. Lett. 57 (1986) 3046.

19. S. Bottin, O. Dauchot, F. Daviaud, Phys. Rev. Lett. 79 (1997) 4377-4380.

20. K. Avila, D.Moxey, A. de Lozar, M.Avila, D. Barkley, B.Hof, Science 333 (2011) 192.

21. S. Fauve, O. Thual, Phys. Rev. Lett. 64 (1990)282-284.

22. O. Stiller, S. Popp, I. Aranson, L. Kramer, Physica D 87 (1995) 361-370.

23. J. Burguete, H. Chate, F. Daviaud, N. Mukolobwiez, Phys. Rev. Lett. 82(1999)32523255 .

24. O. Descalzi, H.R. Brand, Physical Review E 82 (2010) 026203

25. O. Descalzi, C. Cartes, J. Cisternas, H.R. Brand, Physical Review E 83 (2011) 056214

26. C. Cartes, O. Descalzi, H.R. Brand, Physical Review E 85 (2012) 015205

27. C. Cartes, J. Cisternas, O. Descalzi, H.R. Brand, Physical Review Letters 109 (2012) 178303

28. O. Descalzi, H.R. Brand, Phys Rev E 87 (2013) 022915.

29. L. Koshmieder, "Bénard Cells and Taylor Vortices" (Cambridge Univ. Press, Cambridge 1993).

30. A. V. Taraut, B. Smorodin, M. Lücke, New Phys. 14 (2012) 093055. 
31. D. Jung, M. Lücke, Europhys. Lett. 80 (2007) 14002.

32. D. Jung, M. Lücke, Phys. Rev. E 72 (2005) 026307.

33. D. Jung, M. Lücke, Phys. Rev. Lett. 89 (2002) 054502.

34. W. Barten, M. Lücke, M. Kamps. R. Schmitz, Phys. Rev. E51 (1995) 5662.

35. M. Bestehorn, Phys. Lett. A 174 (1993) 43-47. M. Bestehorn, P. Colinet, Physica D 145 (2000) 84-109.

36. P. Kolodner, D. Bensimon, C.M. Surko, Phys. Rev. Lett. 60 (1988) 1723-26

37. C.M. Surko, D.R. Ohlsen, S.Y. Yamamoto, P. Kolodner, Phys. Rev. A 43 (1991) R710104

38. B.I. Winkler, P. Kolodner, J. Fluid Mech. 240 (1992) 31-58

39. P. Kolodner, Phys. Rev. E 50 (1994) 2731-55

40. R. Heinrichs, G. Ahlers, D.S. Cannell Phys. Rev. A 35 (1987) R2761-64

41. K.E. Anderson, R.P. Behringer, Physica 51D (1991) 444-9

42. N. Akhmediev and A. Ankiewicz (eds). Dissipative Solitons Lecture Notes in Physics 661, Spinger, Berlin (2005).

43. M. Dubois, F. Daviaud, O. Ronsin and P. Berge. Physica D 61 (1992) 140-146.

44. J. Burguete, $\mathrm{PhD}$ Thesis "Instabilities in a localized heating experiment" (in spanish, University of Navarra, 1995).

45. J. Burguete, H. Mancini, C. Perez Garca, Europhys. Lett., 23 (1993) 401-407.

46. O. Batiste, E. Knobloch, A. Alonso, I. Mercader, J. Fluid Mech. 560, (2006) 149.

47. A. Alonso, O. Batiste, A. Meseguer, I. Mercader, Physical Rev. E 75 (2007) 026310.

48. A. Bergeon, J. Burke, E. Knobloch, I. Mercader, Physical Review E, 78 (2008) 046201.

49. I. Mercader,A. Alonso, O. Batiste, Physical Review E 77 (2008) 036313.

50. I. Mercader, O. Batiste, A. Alonso, E. Knobloch, Physical Review E 80 (2009) 025201$1 / 7$.

51. I. Mercader, O. Batiste, A. Alonso, E. Knobloch, Fluid Dynamics Research 42 (1994) 025505-1/10.

52. Alonso, A., Batiste, O., Knobloch, E., Mercader, I.. in Localized States in Physics: Solitons and Patterns, Ed. O. Descalzi, M. Clerc, S. Residori and G. Assanto; 109-125, ISBN: 978-3-642-16548-1, Springer-Verlag.

53. I. Mercader, O. Batiste, A. Alonso, E. Knobloch, Discrete and Continuous Dynamical Systems Series S, 4(5), 1213-1225.

54. I. Mercader, O. Batiste, A. Alonso, E. Knobloch, Journal of Fluid Mechanics, 667 (2011) 586-606.

55. M. Lücke, W. Barten, M. Kamps, Physica D 61 (1992) 183-196

56. H. Riecke, W.J. Rappel, Phys Rev Lett 72 (1995) 4035-4038.

57. M. Miranda, "Characterization of thermoconvective instabilities in spatially extended systems"(PhD Thesis, University of Navarra, Pamplona, Spain 2009).

58. H. Herrero, A. M. Mancho, Phys. Rev. E 57 (1998), 7336-7339.

59. A. M. Mancho, H. Herrero, J. Burguete, Phys. Rev. E 56 (1997), 2916-2923.

60. A. Goharzadeh, I. Mutabazi, Physical Review E 82 (2010) 016306.

61. H.R. Brand, R.J. Deissler, Physical Review E, 58 (1998) R4064

62. H.R. Brand, R.J. Deissler, Physical Review A 46 (1992) 888-892.

63. M.A. Miranda, J. Burguete, Physical Review E 78 (2008) 046305

64. M.A. Miranda, J. Burguete, Int Journal Bif Chaos 20 (2008) 835-847

65. M.A. Miranda, J. Burguete, Physical Review E, 79 (2009) 046201

66. M.A. Miranda, J. Burguete, W. Gonzalez-Vinas, H. Mancini, Int Journal Bif Chaos, 22 (2012) 1250165

67. M.A. Miranda, J. Burguete, H. Mancini, W. González-Viñas, Physical Review E 87 (2013) 032902.

68. J.Burguete, N. Mukolobwiez, F. Daviaud, N. Garnier y A. Chiffaudel. Physics of Fluids 13 (2001) pp. 2773-2787.

69. Descalzi, O, private communication. 